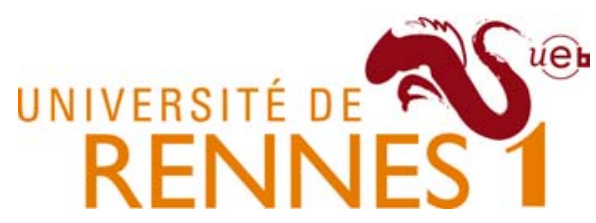

This is the author's final draft post-refeering (post-print)

Find more peer-reviewed articles on our open access repository:

http://hal-univ-rennes1.archives-ouvertes.fr/ 


\title{
How does the electronic continuum model perform in the prediction of the surface tension of salt solutions?
}

\author{
Jean Claude Neyt ${ }^{\mathrm{a}, \mathrm{d}}$, Aurélie Wender ${ }^{\mathrm{a}}$, Véronique Lachet $^{\mathrm{a}}$, Anthony Szymczyk ${ }^{\mathrm{b}}$, Aziz Ghoufi ${ }^{\mathrm{c}}$, \\ Patrice Malfreyt ${ }^{\mathrm{d}, *}$ \\ a IFP Energies nouvelles, 1-4 Avenue de Bois Préau, 92852 Rueil-Malmaison, France \\ ${ }^{\mathrm{b}}$ Institut des Sciences Chimiques de Rennes, UMR CNRS 6226, Université Rennes 1, 35042 Rennes, France \\ ${ }^{\mathrm{c}}$ Institut Physique de Rennes, UMR CNRS 6251, Université Rennes 1, 35042 Rennes, France \\ ${ }^{\mathrm{d}}$ Institut de Chimie de Clermont-Ferrand, ICCF, UMR CNRS 6296, BP 10448, F-63000 Clermont-Ferrand, France
}

\section{A B S T R A C T}

The electronic continuum (EC) model uses a scaling of the charges of the ions in order to model implicitly the polarization into nonpolarizable models. This scaling procedure is applied here to two standard nonpolarizable force fields to investigate the salt concentration dependence of the surface tension and density of $\mathrm{NaCl}$ aqueous solutions. The composition of the interface and the orientation of the water molecules at the water surface are reported for different combinations of force fields.

\section{Introduction}

Whereas Raman and IR measurements [1,2] show that the structure of the liquid-vapour interface of the $\mathrm{NaF}$ and $\mathrm{NaCl}$ salts are similar to that of the pure water, they also establish higher concentrations of bromide and iodide in the interfacial region of $\mathrm{NaBr}$ and $\mathrm{NaI}$ salts. The atomistic description of these interfaces by using atomistic models remains an active field of research [3-8]. It was often suggested that the presence of the anions at the liquid-vapor interface was mainly due to the polarizability [3,4,9-13]. Actually, these atomistic simulations address a number of fundamental questions concerning the choice of the model for considering the polarizability either implicitly $[6,7,14,15]$ or explicitly [16-22]. Three methodologies coexist to consider explicit electronic polarization into atomistic models: induced dipole model [23-25], fluctuation charge model [26,27] and classical Drude oscillator model [17]. However, recent molecular simulations [5,8] were able to reproduce enhancements of iodide density at the water surface by using force fields that do not consider explicitly the polarizability.

A new challenge arises when a quantitative prediction of the surface tension of salt solutions is proposed over a range of concentrations. The modeling of these interfacial systems addresses questions about the dependences of the interfacial properties on both the system-sizes and atomistic models. The force field must

\footnotetext{
* Corresponding author.

E-mail address: Patrice.Malfreyt@univ-bpclermont.fr (P. Malfreyt).
}

then balance the computational efficiency with an reasonable level of accuracy for the microscopic interactions.

Actually, the nonpolarizable models have been shown to be successful in predicting the temperature dependence of the surface tension of many molecular systems [28-30] although these models have been adjusted from only bulk thermodynamic properties. Recently, we have investigated the salt concentration dependence [31] on the surface tension and density of $\mathrm{NaCl}$ aqueous solutions by comparing nonpolarizable models and polarizable models based upon the Drude oscillator formalism. Whereas the Drude polarizable force fields fail to reproduce quantitatively the increase of the surface tension and density with increasing salt concentrations, the atomistic description of the interface shows anions which are closer to the water surface than cations. With some nonpolarizable models, the interface is devoid of ions but the salt concentration dependence of the surface tension is well-reproduced.

Recently, an alternative method was proposed to consider electronic polarization into a simple nonpolarizable model. This methodology is referred to as the electronic continuum (EC) model $[6,32]$ and consists of scaling all the electrostatic interactions by the factor $1 / \epsilon_{e}$ where $\epsilon_{e}$ represents the electronic dielectric constant of the medium. In the case of molecular simulations involving water models such as SPC/E [33] and TIP4P/2005 [34], the phenomenological parameter $\epsilon_{e}$ was taken to $1.78[32,35]$ in order to reproduce the experimental dipole moment of the liquid phase [36]. When salt molecules are considered in aqueous solutions, a question arises about the validity of the value of the scaling factor over a large range of salt concentrations [37]. A new issue appears when this approach is applied to interfacial systems. Indeed, as 
underlined recently by Jungwirth and coworkers [38], this theoretical framework assumes an homogeneous distribution of solvent molecules within each region in the bulk of the same relative permittivity. What about the transferability of this approach to the liquid-vapor interfaces of salt solutions? However, recently, very nice results about the affinity of different anions has been investigated in different types of salt aqueous interfaces such as liquidvapor and water-oil systems [38]. This Letter concludes by an overestimation of the affinity of the ions at the water liquid-vapor interface.

Nevertheless, this rescaling procedure consisting of changing the charges of the ions has already been applied in previous simulations $[12,13]$ of the air-water interface. The distribution of the ions at the interface has been shown to be significantly impacted by the charge of the ion. We propose here to test this rescaling ionic procedure on classical nonpolarizable force fields for the prediction of the salt concentration dependence of the surface tension over a large range of $\mathrm{NaCl}$ concentrations. We take the route of focusing on only the surface tension of $\mathrm{NaCl}$ aqueous solutions because an thorough comparison between polarizable and nonpolarizable models is available for this salt [39]. We also investigate by molecular simulations in bulk conditions the performance of the EC model on the prediction of the density of salt aqueous solutions. We will complement this Letter by a description of the distribution of the ions at the interface in terms of arrangements and orientations.

\section{Model and method}

\subsection{Potential model}

The water is modeled using the TIP4P/2005 description [34]. Two sets of parameters are used for the sodium and chloride ions: one set resulting from the OPLS force-field $[14,21]$ and the other one from the model developed by Reif [7]. The Lennard-Jones parameters and the partial charges of these two ions are given in Table 1. In the EC model, the charges of the ions are scaled by a factor $(1 / \sqrt{\epsilon}) \approx 0.75$ where $\epsilon=1.78$ for water. The total configurational energy sums the intramolecular and intermolecular energy contributions. Since the water TIP4P/2005 is considered as rigid, the intramolecular interactions are zero. The intermolecular interactions are composed of repulsion-dispersion and electrostatic contributions that are represented by Lennard-Jones and Coulombic potentials, respectively. The electrostatic interactions are calculated using the Ewald sum method.

Table 1

The Lennard-Jones well depth $\epsilon$ and size $\sigma$, partial charges $q$ for $\mathrm{Na}^{+}$and $\mathrm{Cl}^{-}$ion models.

\begin{tabular}{|c|c|c|c|}
\hline & $\begin{array}{l}\sigma \\
(\AA)\end{array}$ & $\begin{array}{l}\epsilon / k_{B} \\
(\mathrm{~K})\end{array}$ & $\begin{array}{l}\mathrm{q} \\
(|e|)\end{array}$ \\
\hline \multicolumn{4}{|c|}{ OPLS model $[14,21]$} \\
\hline $\mathrm{Na}^{+}$ & 1.8974 & 808.8 & +1 \\
\hline $\mathrm{Cl}^{-}$ & 4.41724 & 59.27 & -1 \\
\hline \multicolumn{4}{|c|}{$E C+O P L S$} \\
\hline $\mathrm{Na}^{+}$ & 1.8974 & 808.8 & +0.75 \\
\hline $\mathrm{Cl}^{-}$ & 4.41724 & 59.27 & -0.75 \\
\hline \multicolumn{4}{|c|}{ Reif model [7] } \\
\hline $\mathrm{Na}^{+}$ & 4.0813 & 0.4656 & +1 \\
\hline $\mathrm{Cl}^{-}$ & 3.4711 & 217.37 & -1 \\
\hline \multicolumn{4}{|c|}{$E C+$ Reif } \\
\hline $\mathrm{Na}^{+}$ & 4.0813 & 0.4656 & +0.75 \\
\hline $\mathrm{Cl}^{-}$ & 3.4711 & 217.37 & -0.75 \\
\hline
\end{tabular}

\subsection{Simulation method}

The equation of motions are solved using the Nosé-Hoover algorithm using $0.1 \mathrm{ps}$ for the thermostat relaxation time with a time step of $1 \mathrm{fs}$. The initial simulation box is a rectangular parallelepipedic box of dimensions $L_{x} L_{y} L_{z}\left(L_{x}=L_{y}=40 \AA\right)$ formed by water and ions. The total number of water molecules was fixed to 2000 and the number of $\mathrm{Na}^{+}$and $\mathrm{Cl}^{-}$ions was increased from 20 to 200 over the range of molalities investigated here. After some of equilibration cycles under constant-NpT conditions, the box was elongated along the $z$-direction with $L_{z}=120 \AA$ A to create two interfacial regions. Periodic boundary conditions are applied in the three directions. Standard deviations of the ensemble averages are calculated by breaking the production runs into block averages. The calculated number of block averages is adjusted in order to allow the convergence of the surface tension within each block. The cutoff radius is set to $12 \AA$. The systems are equilibrated for $2 \mathrm{~ns}$ followed by a production phase in the range of $5-10 \mathrm{~ns}$.

\subsection{Surface tension calculation}

The methods used here for the surface tension calculation are based upon the mechanical route definition and use the tensorial components of the pressure. The first explicit form expresses the components of the pressure tensor as a function of the derivative of the intermolecular potential. This operational expression was given by Kirkwood and Buff [40] and is referred here as the KB expression $\left(\gamma_{\mathrm{KB}}\right)$. The definition of Irving and Kirkwood [41] $\left(\gamma_{\mathrm{IK}}\right)$ is based upon the notion of the force across a unit area and takes advantage of expressing the local components of the pressure tensor along the direction normal to the surface. A novel method based upon the thermodynamic definition of the surface tension $\left(\gamma_{\mathrm{TA}}\right)$ has been established by Gloor et al. [42] and consists in perturbing the cross-sectional area of the system [43] containing the interface. $\left(\gamma_{\mathrm{Kвz}}\right)$ can be interpreted as a local version of the KB version. Due to the truncation of the Lennard-Jones potential, the surface tension must be corrected by specific long range corrections (LRC). The operational expressions of these LRC contributions are also given in Ref. [44] for the IK, KB, KBZ and TA approaches. For multicomponent systems such as salts, the operational expression of $\gamma_{\text {KB,LRC }}$ is not longer valid. As a result, we take the route of replacing this contribution by that of $\gamma_{\mathrm{KBZ}}$.

\section{Results and discussions}

NpT MD bulk simulations of the $\mathrm{NaCl}$ solutions has been carried out over a molality range of $0.5-5.5 \mathrm{~mol} \mathrm{~kg}^{-1}$ at $0.1 \mathrm{MPa}$ and $298 \mathrm{~K}$. The charges of the sodium and chloride ions are scaled by 0.75 in the OPLS and Reif models in line with the implicit scaling factor of the TIP4P/2005 model. The resulting calculated densities are reported as a function of the salt concentration in Figure 1. For comparison, we report the densities [39] calculated from the original ion models. First, we observe that all models predict an increase of the density with the salt concentration. Second, decreasing the magnitude of the charges of the ions amounts to underestimating the aqueous $\mathrm{NaCl}$ densities. Whereas the best agreement with experiments is obtained with the OPLS model that gives an average deviation of $0.2 \%$ over the range of concentrations, the scaling procedure applied to this model leads to an average deviation of $4 \%$ from experiments. For the Reif model, the simulated densities are overestimated with respect to experiments with an average deviation of $2.2 \%$. For the EC version of the Reif model, the average deviation from experiments is about the same $(2.5 \%)$ but now the calculated densities are underestimated. 


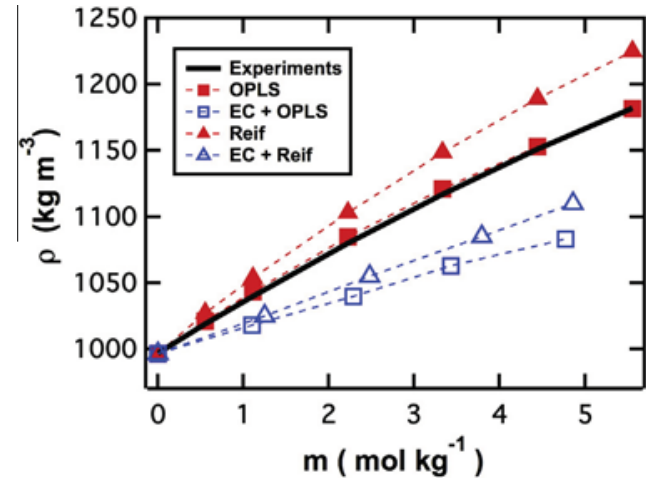

Figure 1. Densities of $\mathrm{NaCl}$ solutions calculated using NPT MD simulations as a function of the molal concentration of $\mathrm{NaCl}$. The experimental densities [45] are represented by the solid black line. The statistical fluctuations, that correspond to about $0.5 \%$ of the simulated density, are smaller than the size of the different symbols. The different models for the ions are indicated in the legend.

We now turn to the calculation of the surface tension of $\mathrm{NaCl}$ solutions over a large range of molalities. We report for comparison in Figure 2 the surface tensions calculated from the standard OPLS and Reif models. We also plot the experimental surface tensions calculated from the $\frac{d \gamma}{d m}$ surface tension increments [9]. The surface tensions are reported in Table 2 with the long range corrections for the thermodynamic and mechanical definitions at different salt concentrations. First, the standard nonpolarizable models give a linear dependence of the surface tension on the molality. Linear regressions give slopes of $2.0 \pm 0.4$ and $1.5 \pm 0.2$ for the Reif and OPLS models, respectively. These slopes compare very well with the corresponding experimental values of 1.7 and 1.9 [9] $\mathrm{mN} \mathrm{m}^{-1} \mathrm{~mol}^{-1} \mathrm{~kg}$. Additionally, the maximum deviation from experiments is about $4.5 \%$ and $7 \%$ with the OPLS and Reif models, respectively. Such a linear dependence on salt concentrations has been already reproduced for the $\mathrm{NaF}$ salt by atomistic simulations using nonpolarizable models [5]. Second, when the EC correction is applied to the nonpolarizable models, we observe that the simulations are unable to reproduce a monotonic increase of the surface tension with the salt concentration. A maximum

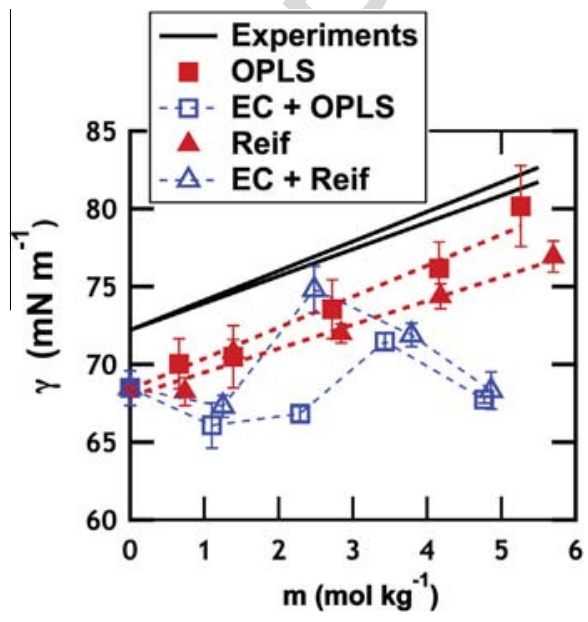

Figure 2. Surface tensions as a function of the molal concentration of $\mathrm{NaCl}$ for the OPLS and Reif models and EC versions of these models as illustrated in the legend. The surface tension is averaged over the KB, KBZ, IK and TA methods. We also plot for comparison the experimental surface tension increment $\left(\frac{d \gamma}{d m}\right)$ that defines the slope of the linear dependence of the surface tension on molality [9]. The dotted lines (red) represent the surface tension increments calculated from linear fits of the calculated surface tensions. (For interpretation of the references to colour in this figure legend, the reader is referred to the web version of this article.)
Table 2

Surface tension values $\left(\mathrm{mN} \mathrm{m}^{-1}\right)$ of the liquid-vapour interface of salt solutions as a function of the $\mathrm{NaCl}$ molal concentration $m\left(\mathrm{~mol} \mathrm{~kg}^{-1}\right)$. The long range corrections $\left(\gamma_{\text {LRC }}\right)$ and the total surface tension $(\gamma)$ are given for each method. $\langle\gamma\rangle$ is averaged over the IK, KB, TA and KBZ approaches. The subscripts give the accuracy of the last decimal (s), i.e., $68.4_{11}$ means $68.4 \pm 1.1$.

\begin{tabular}{|c|c|c|c|c|c|c|c|c|c|}
\hline \multirow[t]{2}{*}{$\mathrm{m}$} & \multicolumn{2}{|l|}{$\gamma_{\mathrm{KB}}$} & \multicolumn{2}{|l|}{$\underline{\gamma}_{\mathrm{IK}}$} & \multicolumn{2}{|l|}{$\gamma_{\mathrm{TA}}$} & \multicolumn{2}{|l|}{$\gamma_{\mathrm{KBZ}}$} & \multirow[b]{2}{*}{$\langle\gamma\rangle$} \\
\hline & $\gamma_{\text {LRC }}$ & $\gamma$ & $\gamma_{\text {LRC }}$ & $\gamma$ & $\gamma_{\text {LRC }}$ & $\gamma$ & $\gamma_{\text {LRC }}$ & $\gamma$ & \\
\hline \multicolumn{10}{|c|}{$E C+O P L S$} \\
\hline 0 & $1.3_{1}$ & $68.2_{11}$ & $1.5_{1}$ & $68.6_{11}$ & $1.1_{1}$ & $68.6_{11}$ & $1.3_{1}$ & $68.4_{11}$ & $68.4_{11}$ \\
\hline 1.1 & $1.4_{1}$ & $65.8_{15}$ & $1.6_{1}$ & $66.3_{15}$ & $1.0_{1}$ & $66.3_{15}$ & $1.4_{1}$ & $66.0_{15}$ & $66.1_{15}$ \\
\hline 2.29 & $1.4_{1}$ & $66.6_{5}$ & $1.7_{1}$ & $67.0_{5}$ & $1.1_{1}$ & $67.0_{5}$ & $1.4_{1}$ & $66.7_{5}$ & $66.8_{5}$ \\
\hline 3.43 & $1.4_{1}$ & $71.2_{5}$ & $1.7_{1}$ & $71.7_{5}$ & $1.1_{1}$ & $71.6_{5}$ & $1.4_{1}$ & $71.4_{5}$ & $71.5_{5}$ \\
\hline 4.77 & $1.5_{1}$ & $67.4_{2}$ & $1.8_{1}$ & $67.9_{2}$ & $1.1_{1}$ & $67.9_{2}$ & $1.5_{1}$ & $67.6_{2}$ & $67.7_{2}$ \\
\hline \multicolumn{10}{|c|}{$E C+$ Reif } \\
\hline 0 & $1.3_{1}$ & $68.2_{11}$ & $1.5_{1}$ & $68.6_{11}$ & $1.1_{1}$ & $68.6_{11}$ & $1.3_{1}$ & $68.4_{11}$ & $68.4_{11}$ \\
\hline 1.25 & $1.3_{1}$ & $67.0_{7}$ & $1.6_{1}$ & $67.5_{7}$ & $1.0_{1}$ & $67.5_{7}$ & $1.3_{1}$ & $67.2_{7}$ & $67.3_{7}$ \\
\hline 2.48 & $1.4_{1}$ & $75.5_{15}$ & $1.6_{1}$ & $75.0_{15}$ & $1.0_{1}$ & $75.0_{15}$ & $1.4_{1}$ & $74.7_{15}$ & $74.8_{15}$ \\
\hline 3.79 & $1.4_{1}$ & $71.6_{8}$ & $1.6_{1}$ & $72.1_{8}$ & $1.0_{1}$ & $72.1_{8}$ & $1.4_{1}$ & $71.8_{8}$ & $71.9_{8}$ \\
\hline 4.86 & $1.6_{1}$ & $68.0_{12}$ & $1.6_{1}$ & $68.5_{12}$ & $1.0_{1}$ & $68.5_{12}$ & $1.6_{1}$ & $68.2_{12}$ & $68.3_{12}$ \\
\hline
\end{tabular}

deviation from experiment of $17 \%$ is even obtained with the EC correction at the highest molalities. This is exactly what we observe with the polarizable models based on the Drude oscillator model [31] for which the surface tension are found to increase non-monotonically with the salt concentration. This evidences the impact of the scaling procedure of the charges on the prediction of the surface tension over a large range of concentrations. A better reproduction of the surface tension over this concentration range would require a combination of the charge scaling procedure with a consistent re-parameterization of the nonpolarizable force fields.

In order to dismiss a lack of convergence of the liquid-vapor equilibrium, we plot in Figure 3 the normal and tangential components of the pressure tensor along the $z$ direction normal to the surface. The kinetic (ideal-gas) term of the pressure $\rho(z) k_{B} T$ is the same in the normal and tangential components. As a result, it has been omitted for clarity in Figure 3. We also plot the profiles of the difference between the normal and tangential components of the pressure tensor and of the integral of this difference $\gamma(z)=\int_{-L_{z} / 2}^{z}\left(p_{N}\left(z_{k}\right)-p_{T}\left(z_{k}\right)\right) d z_{k}$ along the direction normal to the interface. These profiles correspond to well-equilibrated twophase configurations with two symmetric $p_{N}-p_{T}$ peaks around the middle of the box: $p_{T}(z)$ must be negative at the interfaces. The bulk phases are well-developed and they do not contribute to the surface tension: $p_{N}(z)=p_{T}(z)$ in the bulk phases. The

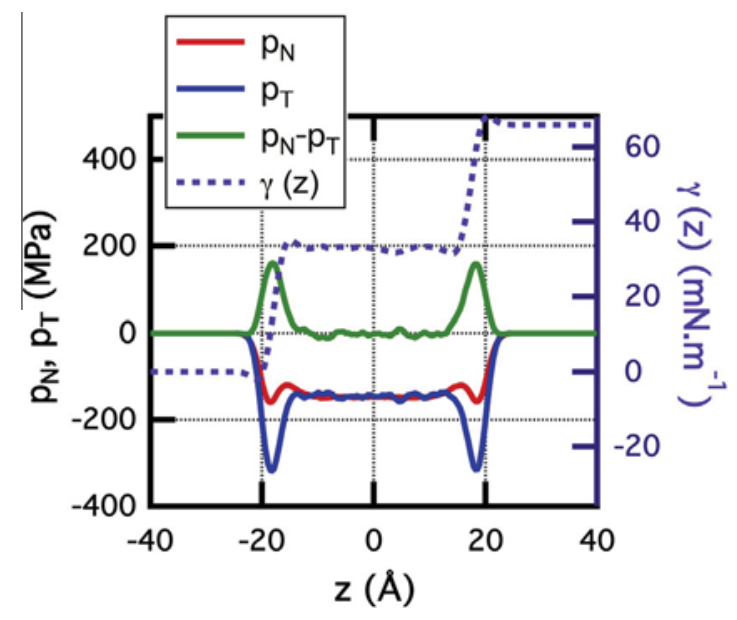

Figure 3. Profiles of the configurational part of the normal $\left(p_{N}(z)=p_{z z}(z)\right)$ and tangential $\left(p_{T}(z)=1 / 2\left(p_{x x}(z)+p_{y y}(z)\right)\right.$ of the pressure tensor. The difference $p_{N}(z)-p_{T}(z)$ is also represented with its integral $\gamma(z)$ (right axis). 

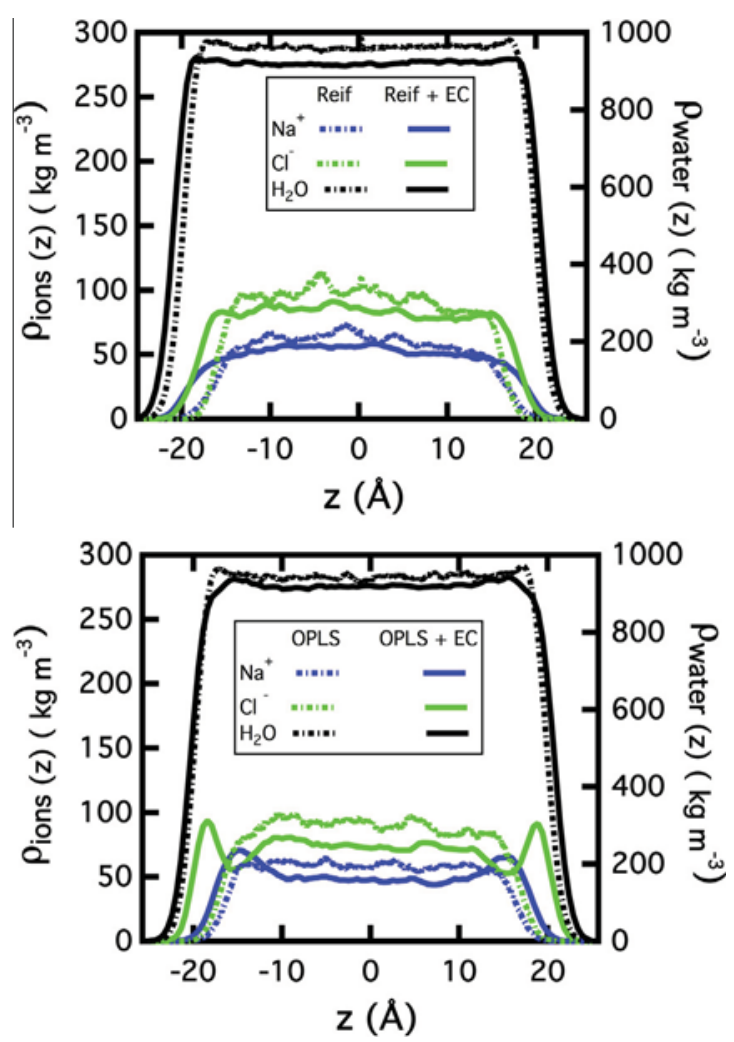

Figure 4. Density profiles along the $z$ direction for the water molecules (right axis), sodium and chloride ions (left axis) as indicated in the legend. Top panel: Reif model and bottom panel: OPLS model at a molality of about $2 \mathrm{~m}$.

contribution to $\gamma(z)$ from both surfaces is the same. These profiles show very well-equilibrated two phase configurations as required by the mechanical equilibrium of these planar interfaces.

The density profiles of the salt ions and water are given in Figure 4. When the standard nonpolarizable Reif and OPLS models are considered, the composition of the interfacial region is the same: the ions are excluded from the liquid-vapour interface and the profiles of the chloride anions and sodium cations are similar. These models reproduce then the long-standing picture of an interface devoid of ions [46]. However, this traditional picture was challenged by recent molecular simulations using polarizable models $[4,31,47,48]$, theory $[10,49]$ and experiments $[2,50]$. Interestingly, when the charge scaling procedure is applied to these models, the structure at the interface changes significantly. For the (Reif + EC) model, we observe that the two ions populate the interfacial region near the water surface and the density profiles of $\mathrm{Na}^{+}$ and $\mathrm{Cl}^{-}$remain qualitatively similar. For the (OPLS + EC) model, the effect is still more pronounced: we observe a preferential adsorption of anions at the water surface whereas sodium cation is repelled from the surface. This enhancement of anion concentration is followed by a region of density depletion of anions that corresponds to an excess in the sodium concentration. This double ionic layer structure develops an electric field that may impact significantly the orientation of water molecules in this zone. The description of the interface with the OPLS + EC model agrees qualitatively with what is expected from the introduction of polarizability for ions at the aqueous interface $[4,47]$ even if the ionic charge separation at the water surface is exaggerated by the scaling procedure. We also observe that the scaling procedure does affect the composition of the interfacial region with arrangements of ions dependent on the force field. It is interesting to note from previous molecular simulations using nonpolarizable models $[5,8]$ that the enhancement of anion density at the interface can be obtained by increasing the size of the anion.

The orientational structure of the water molecules in the interfacial region is illustrated in Figure 5 through the distribution of the angle between the water dipole vector and the vector normal to the surface. First, Figure 5 shows there is a broad range of possible orientations in the interfacial region whereas this angle distribution (not shown here) is flat in isotropic bulk environment. In the case of a liquid-vapour interface of a pure water system, the preferential orientation places the water molecular plane parallel to the interface. The presence of salt reorients the water molecules with an increase of the population of angles greater than $140^{\circ}$ : as a result, the water molecules point their dipole moment toward the

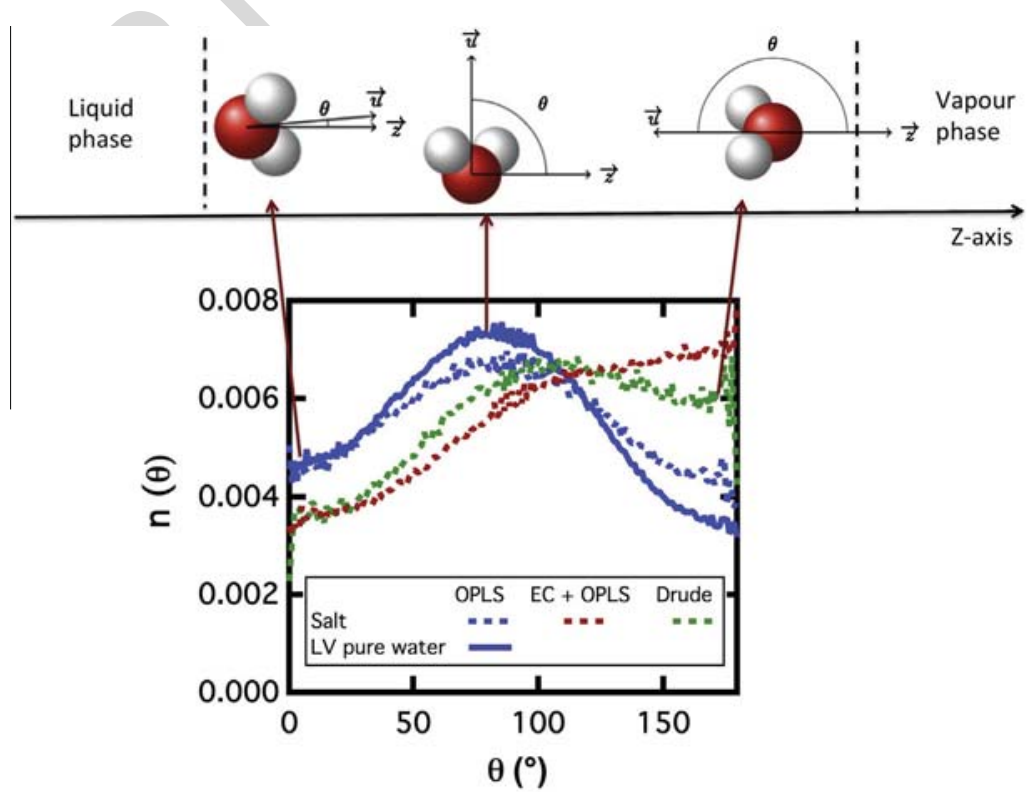

Figure 5. Distribution of the $\theta$ angle between the molecular dipole vector of water and the vector normal to the surface at about $3 \mathrm{~m}$. $\theta=0^{\circ}$ means that the water molecules point their hydrogen atoms toward the vapor phase whereas they direct their dipoles toward the liquid phase for $\theta=180^{\circ}$. Distributions of angle close to $90^{\circ}$ indicate that the symmetry axis of water molecules lies parallel to the surface as indicated in the legend. Concerning the Drude oscillator model, water and ions are represented using the Drude oscillator SW4-NDP and AH-NDP models [31]. 
bulk liquid phase. The number of such orientations is increasing from the Drude oscillator model to the electronic continuum in response to the double electric layer formation. This agrees very well with the fact that the ionic double layer is much more marked with the EC correction.

\section{Summary}

Molecular simulations of $\mathrm{NaCl}$ aqueous liquid-vapour interfaces have been performed with the electronic continuum model in order to evaluate the impact of this correction of the prediction of salt concentration dependence on the density and surface tension. Whereas the original version of the nonpolarizable models exhibits quantitative salt concentration dependence of the surface tension, the electronic continuum correction is unable to reproduce a monotonic increase of the surface tension with the molality. This inability of reproducing the surface tension and the liquid density at different salt concentrations cannot be attributed to a poor convergence of the two-phase simulations that exhibit profiles of pressure components in line with the expected mechanical equilibrium of planar interfaces. Whereas the nonpolarizable models give a picture of an interface devoid of ions, the electronic continuum model tends to migrate the ions toward the water surface: the extent of this migration depends on the force field used. The combination of the OPLS and EC models leads to the formation of an ionic double layer in line with what is observed with polarizable models. The water molecules align themselves with the electric field generated by the ionic double layer: they point their dipoles toward the bulk liquid phase.

\section{References}

[1] D. Liu, G. Ma, L.M. Levering, H.C. Allen, J. Phys. Chem. B 108 (2004) 2252.

[2] E.A. Raymond, G.L. Richmond, J. Phys. Chem. B 108 (2004) 5051.

[3] P. Jungwirth, D.J. Tobias, J. Phys. Chem. B 105 (2001) 10468.

[4] P. Jungwirth, D.J. Tobias, J. Phys. Chem. B 106 (2002) 6361.

[5] D.J.V.A. dos Santos, F. Müller-Plathe, V.C. Weiss, J. Phys, Chem. C 112 (2008) 19431.

[6] I. Leontyev, A. Stuchebrukhov, J. Chem. Phys. 130 (2009) 085102.

[7] M.M. Reif, P.H. Hünenberger, J. Chem. Phys. 134 (2011) 144104.

[8] F. Bresme, E. Chacón, P. Tarazona, A. Wynveen, J. Chem. Phys. 137 (2013) 114706.
[9] L.M. Pegram, J.M.T. Record, J. Phys. Chem. B 111 (2007) 5411.

[10] Y. Levin, A dos Santos, A. Diehl, Phys. Rev, Lett. 103 (2009) 257802

[11] D. Horinek, A. Herz, L. Vrbka, F. Sedlmeier, S.I. Mamatkulov, R.R. Netz, Chem. Phys. Lett. 479 (2009) 173.

[12] O.T. Cummings, C.D. Wick, Chem. Phys. Lett. 500 (2010) 41.

[13] D.E. Otten, P.R. Shaffer, P.L. Geissler, R.J. Saykally, Proc. Natl. Acad. Sci. USA 109 (2012) 701.

[14] J. Chandrasekhar, D.C. Spellmeyer, W.L. Jorgensen, J. Am. Chem. Soc. 106 (1984) 903

[15] D.R. Wheeler, J. Newman, J. Phys. Chem. B 108 (2004) 18353.

[16] J.W. Caldwell, P.A. Kollman, J. Phys. Chem. 99 (1995) 6208.

[17] G. Lamoureux, J.A.D. MacKerell, B. Roux, J. Chem. Phys. 119 (2003) 5185.

[18] G. Lamoureux, E. Harder, I.V. Vorobyov, B. Roux, J.A.D. MacKerell, Chem. Phys. Lett. 418 (2006) 245.

[19] T. Ishiyama, A. Morita, J. Phys. Chem. C 111 (2007) 721.

[20] G.L. Warren, S. Patel, J. Phys. Chem. C 112 (2008) 7455.

[21] R. D’Auria, D.J. Tobias, J. Phys. Chem. A 113 (2009) 7286

[22] L. Viererblova, J. Kolafa, Phys. Chem. Chem. Phys. 13 (2011) 19925.

[23] P.T. van Duijnen, M. Swart, J. Phys. Chem. A 102 (1998) 2399.

[24] S.Y. Noskov, G. Lamoureux, B. Roux, J. Phys. Chem. B 109 (2005) 6705.

[25] D. Elking, T. Darden, R.J. Woods, J. Comput. Chem. 28 (2007) 1261.

[26] J. Gasteiger, M. Marsili, Tetrahedron Lett. (1978) 3181.

[27] A.K. Rappe, W.A. Goddard, J. Phys. Chem. 95 (1991) 3358.

[28] A. Ghoufi, F. Goujon, V. Lachet, P. Malfreyt, Phys. Rev. E 77 (2008) 031601.

[29] J.C. Neyt, A. Wender, V. Lachet, P. Malfreyt, J. Phys. Chem. B 115 (2011) 9421.

[30] J.C. Neyt, A. Wender, V. Lachet, P. Malfreyt, J. Phys. Chem. C 116 (2012) 10563.

[31] J.C. Neyt, A. Wender, V. Lachet, A. Ghoufi, P. Malfreyt, J. Chem. Phys. 139 (2013) 024701.

[32] I. Leontyev, A. Stuchebrukhov, Phys. Chem. Chem. Phys 13 (2011) 2613.

[33] H.J.C. Berendsen, J.R. Grigera, T.P. Straatsma, J. Phys. Chem. 91 (1987) 6269.

[34] J.L.F. Abascal, C. Vega, J. Chem. Phys. 123 (2005) 234505.

[35] I.V. Leontyev, M.V. Vener, I.V. Rostov, M.V. Basilevsky, M.D. Newton, J. Chem. Phys. 119 (2003) 8024.

[36] Y.S. Badyal, M.-L. Saboungi, D.L. Price, S.D. Shastri, D.R. Haeffner, A.K. Soper, J. Chem. Phys. 112 (2000) 9206.

[37] J.B. Hasted, D.M. Ritson, C.H. Collie, J. Chem. Phys. 16 (1948) 1.

[38] M. Vazdar, E. Pluharova, P.E. Mason, R. Vacha, P. Jungwirth, J. Phys. Chem. Lett. 3 (2012) 2087.

[39] J.C. Neyt, A. Wender, V. Lachet, A. Ghoufi, P. Malfreyt, Phys. Chem. Chem. Phys 15 (2013) 11679.

[40] J.G. Kirkwood, F.P. Buff, J. Chem. Phys. 17 (1949) 338.

[41] J.H. Irving, J.G. Kirkwood, J. Chem. Phys. 18 (1950) 817.

[42] G.J. Gloor, G. Jackson, F.J. Blas, E. de Miguel, J. Chem. Phys. 123 (2005) 134703.

[43] A. Ghoufi, P. Malfreyt, Mol. Phys. 104 (2006) 2929.

[44] F. Biscay, A. Ghoufi, V. Lachet, P. Malfreyt, J. Phys. Chem. B 113 (2009) 14277.

[45] A.M. Rowe, J.C.S. Chou, J. Chem. Eng. Data 15 (1970) 61.

[46] L. Onsager, N.N.T. Samaras, J. Chem. Phys. 2 (1934) 528.

[47] P. Jungwirth, D.J. Tobias, Chem. Rev. 106 (2006) 1259.

[48] B.A. Bauer, S. Patel, J. Chem. Phys. 132 (2010) 024713.

[49] Y. Levin, Phys. Rev. Lett. 102 (2009) 147803.

[50] S. Ghosal et al., Science 307 (2005) 563. 